\title{
Emotion regulation in bipolar disorder: Are emotion regulation abilities less compromised in euthymic bipolar disorder than unipolar depressive or anxiety disorders?
}

\author{
Rodrigo Becerra $^{1 *}$, Kate Cruise ${ }^{1}$, Greg Murray ${ }^{2}$, Darryl Bassett ${ }^{3,4}$, Craig Harms ${ }^{1}$, Alfred Allan ${ }^{1}$, \\ Sean Hood $^{4}$ \\ ${ }^{1}$ School of Psychology, Edith Cowan University, Perth, Australia \\ ${ }^{2}$ Psychological Sciences and Statistics, Swinburne University of Technology, Melbourne, Australia \\ ${ }^{3}$ School of Medicine, University of Notre Dame, Fremantle, WA Australia and Psychiatrist in Private Practice, Perth, Australia \\ ${ }^{4}$ School of Psychiatry \& Clinical Neurosciences (M521), University of Western Australia, Perth, Australia; \\ Email: ${ }^{*}$.becerra@ecu.edu.au
}

Received 3 June 2013; revised 5 July 2013; accepted 18 August 2013

Copyright (c) 2013 Rodrigo Becerra et al. This is an open access article distributed under the Creative Commons Attribution License, which permits unrestricted use, distribution, and reproduction in any medium, provided the original work is properly cited.

\begin{abstract}
Objective: This study investigated the profile of emotion dysregulation in Bipolar Disorder (BD) and compared it to Unipolar Depression, Anxiety, and Healthy control groups. Methods: 148 euthymic patients diagnosed with BD $(n=48)$, Unipolar Depressive disorder $(n=50)$, Anxiety disorder $(n=50)$, and a Healthy Control (HC) group $(n=48)$ were evaluated using the Difficulties in Emotion Regulation Scale (DERS). The DERS yields a total score in addition to scores on six subcomponents believed to encapsulate the emotion dysregulation construct. Results: Compared to the healthy control group, all clinical groups (BD, Unipolar Depression, and Anxiety) reported significantly greater overall difficulties in emotion regulation (Total DERS) and difficulties specific to the DERS subcomponent measures: Goals, Impulse, and Strategies. The profile of emotion dysregulation was virtually identical for the Unipolar Depression and Anxiety groups, with BD demonstrating emotion regulation difficulties intermediate between controls and the two clinical groups. Specifically, emotion regulation in the BD group was significantly less compromised in the domains of acceptance of emotions, emotional awareness, and emotional clarity compared to the depression and anxiety groups. Conclusions: Emotion regulation abilities among people with euthymic BD were significantly less compromised than Unipolar Depression and Anxiety groups with regards to emotional awareness, acceptance of emotions, and understanding of emotions. However, emo-
\end{abstract}

${ }^{*}$ Corresponding author. tion regulation abilities pertaining to engagement in goal directed behaviour, impulse control, and access to emotion regulation strategies were similarly compromised across all three clinical groups. This profile might help enrich extant adjunct psychological interventions for BD by enlisting emotion regulation strategies with the aim of decreasing the relapse rate that characterises BD.

Keywords: Bipolar; Euthymic; Remission; Emotion Dysregulation; Depression; Anxiety; Transdiagnostic

\section{INTRODUCTION}

Bipolar Disorder (BD) is a chronic psychiatric condition characterised by manic, depressive and mixed states (American Psychiatric Association, 2000). In spite of significant advances in its treatment, the course of BD is typically characterised by high rates of relapse and hospitalisation. For example, a seminal longitudinal study found that $37 \%$ of patients taking mood stabilizing medications relapsed within one year, $60 \%$ in two years, and $73 \%$ in five or more years [1]. Moreover, psychosocial functioning and quality of life often remain impaired between mood episodes [2-4]. The burden on individuals, their families and health care systems is therefore significant [5]. Several factors are recognised to trigger mood episodes including psychosocial challenges and their emotional consequences [6]. Surprisingly, there is little research on the emotional strategies that people with $\mathrm{BD}$ use to deal with psychosocial challenges. In particular, little is known about the emotion regulation (ER) profile associated with BD. 
Emotion regulation (ER) refers to our attempts to influence the type of emotions we experience, how we experience them, and how they are expressed. At its most basic level, ER is defined as any attempt to influence the emotions which are experienced and the way in which they are expressed [7]. Deficits in the skills required for ER (emotion dysregulation) impact significantly on psychosocial function and underlie a number of psychopathologies [7]. The current emphasis of ER in a clinical setting rejects earlier assumptions that regulation must involve "control" or "reduction" of emotional arousal [8]. On the contrary, experiencing the so called negative emotions in particular could be part of a healthy regulatory coping system. Response focused ER occurs once a particular emotion has been initiated and concerns the individuals attempts to modulate their response tendencies [7]. Compared to emotion regulation processes that occur at the level of appraisal (i.e., antecedent-focused) [7] regulation abilities that occur at the response level, once an emotion has been activated and modulation processes are initiated, are particularly amenable to clinical intervention.

In response to the absence of an adequate measure of ER, Gratz and Roemer [9] developed the Difficulties of Emotion Regulation Scale (DERS). The conceptual underpinning of the development of this scale lies in the behavioural theories of psychopathology that emphasise the function of problem behaviours and de-emphasises symptom picture [9]. The DERS conceptualises ER from a response tendency perspective, defining ER as involving 1) awareness and understanding of emotions, 2) acceptance of emotions, 3) ability to control impulsive behaviours, 4) ability to meet desired goals whilst experiencing negative emotions, and 5) ability to use situationally appropriate ER strategies to meet desired goals and to meet situational demands [9].

Previous clinical research involving the DERS has indicated that some aspects of ER might be shared among various diagnostic categories. For example, deficits in the attainment of goals (Goals; i.e., concentrating and accomplishing tasks when experiencing negative emotions), impulsivity (Impulse; i.e., remaining in control of behaviour when experiencing negative emotions), and access to regulation strategies (Strategies; i.e., belief that once experiencing a negative emotion, little can be done to regulate that emotion) are common among depressed populations [10,11] and anxious populations [12-14]. Deficits in Clarity (i.e., understanding of the type of emotion experienced) and non-acceptance of emotions (Non-acceptance; i.e., tendency towards secondary negative emotions) have been identified among anxious populations [12-14] but not among depressed populations [10,11]. One recent study [15] investigated cognitiveemotional regulation processes among people with BD in the context of three emotionally evocative films (neutral, happy, sad). Participants made greater use of spontaneous suppression and reappraisal across all films compared to the healthy control group and also reported greater effort but less success, when spontaneously regulating emotions compared to the control group. Currently, no studies identified to date have investigated ER profiles in BD using a clinically relevant or "functional" ER measure such as the DERS.

One could argue that a more clinical approach to the understanding of emotional functioning of persons diagnosed with BD should focus on the context of adaptive abilities and the way emotions are regulated, or otherwise, in response to challenges in the environment of people with BD. No studies to date have simultaneously compared the ER profiles of people with a diagnosis of Unipolar Depression and Anxiety. Moreover, previous research has not consistently investigated all six subscales of the DERS (Non-acceptance, Goals, Awareness, Impulsivity, Strategies, and Clarity), rather selecting a limited number of scales for investigation (e.g., [10,14, 16,17]).

\section{The Present Study}

First, the overarching aim of the present study was therefore to create a profile of ER processes among euthymic $\mathrm{BD}$ and compare this profile to euthymic Unipolar Depression, euthymic Anxiety disorder, and Healthy controls. We sought to identify ER processes that were unique to $\mathrm{BD}$, as well as those that are shared across the disorder groupings. Current taxonomies imply that BD and Unipolar Depression (being diagnostically grouped as Mood Disorders) would share more commonalities than Anxiety Disorders. However, it is not entirely clear what pattern of results might be observed among diagnostic groups as exploring these three psychiatric diagnoses utilising a functional ER parameter has not been reported in the literature.

\section{METHOD}

\subsection{Participants}

Between February 2008 and May 2012, 148 patients diagnosed with a bipolar disorder $(n=48)$ a Unipolar disorder $(n=50)$, or an Anxiety disorder $(n=50)$ were recruited from a private Psychiatrist (author DB), clinical psychologist (author RB), and as part of an ongoing neuro-psychological predictors of recovery from mood episodes in BD study (Edith Cowan University). Fortyeight healthy control (HC) participants were recruited from the community. Participants were aged between 19 and 68 years. Approval was obtained from the Edith Cowan University, Human Research Ethics Committee, and the Southern Metropolitan Area Health Service (SMAHS) Human Research Ethics Committee, Western 
Australia.

Amongst the clinical sample, inclusion criteria were a diagnosis of either (BD Type 1 or Type 2), Unipolar Depressive disorder, and Anxiety disorder (generalised anxiety disorder, phobic anxiety disorder, anxiety disorder not otherwise specified) via the International Classification of Diseases version 10 (ICD-10). All participants recruited via author DB and author RB were assessed as being in a clinically stable state (these patients had access to group therapy once their psychiatric review deemed they have reached a euthymic stage). In addition to participants recruited via $\mathrm{DB}$ (private psychiatrist) and $\mathrm{RB}$ (private psychologist) fourteen participants with $\mathrm{BD}$ were recruited from the community as part of the ongoing neuropsychological predictors of recovery in BD study. These participants were assessed as being within the normal to mild range for depressive symptoms via the Hamilton Depression Ratings Scale (scores 0 - 13) [18] and were not currently experiencing a manic or hypomanic episode (indicated by scores $<5$ on the Young Mania Rating Scale) [19].

\subsection{Medication}

Forty-seven subjects in this study were taking psychotropic medications at the time of the assessment. Ninety one per cent were taking at least one mood stabilizer (34\% lithium and 34\% sodium valproate, with a smaller number either carbamazepine or lamotrigine), fifty three per cent an antipsychotic medication (notably quetiapine but also olanzapine, risperidone, amisulpride and clozapine), forty five per cent an antidepressant (venlafaxine, sertraline, paroxetine, fluoxetine, duloxetine, clomipramine and escitalopram), and twenty one per cent a benzodiazepine (lorazepam, clonazepam).

\subsection{Materials and Procedure}

Participants completed the Difficulties in Emotion Regulation Scale (DERS) [9]. The DERS assesses both general deficits in ER and deficits in specific domains of ER. The DERS assesses difficulties in ER during times of distress; therefore many items begin with "When I am upset”. Difficulties in emotion regulation are assessed via six subscales: 1) non acceptance of emotions [NonAcceptance] (e.g., "When I am upset, I feel guilty for feeling that way"); 2) difficulties in engaging in goal directed behaviour when distressed [Goals] (e.g., "When I'm upset, I have difficulty concentrating); 3) impulse control difficulties [Impulse] (e.g., "When I'm upset, I become out of control); 4) lack of emotional awareness [Awareness] (e.g., "I pay attention to how I feel [reversed]); 5) limited access to emotion regulation strategies [Strategies] (e.g., "When I'm upset it takes me a long time to feel better); 6) lack of emotional clarity [Clarity] ("I am confused about how I feel"). Higher scores indicate greater difficulties in emotion regulation Participants are required to indicate how often each items apply to themselves, with responses ranging from 1 to 5 , where 1 is almost never $(0 \%-10 \%) 2$ is sometimes $(11 \%$ - 35\%) 3 is (about half the time $36 \%-65 \%$ ) 4 is most of the time $(66 \%-90 \%)$ and 5 is almost always $(91 \%$ $100 \%$ ). The DERS demonstrates good psychometric properties for all subscales $(\alpha$ 's $>0.80)$ [9]. Good to excellent reliability was also demonstrated for all DERS subscales and total scores for the current study (Chronbach $\alpha$ non acceptance $=0.921$; goals $=0.906$; awareness $=0.751 ;$ impulse $=0.903 ;$ strategies $=0.794$; clarity $=$ 0.855; total DERS $=0.903$ ).

\subsection{Statistical Analyses}

Mean substitution was used to impute missing values when calculating subscale scores. Total scores for subscales involving more than one missing value were excluded from analyses Forty values were excluded from analysis in total (Non-Acceptance $=10$; Goals $=10$; Impulse $=10$; Strategies $=10$ ). All total DERS subscale scores included no more than six missing values and were therefore included in the analysis. The four groups (BD, Depression, Anxiety, and Healthy controls) were compared on age, the six DERS subscales and total DERS using one-way analysis of variance (ANOVA) and Chi-Square analyses were used to inspect for differences in gender composition between groups.

Tukey HSD post hoc tests were used to test for significant differences between individual means where main effects were significant. Statistical significance was set at $p<0.05$ and SPSS 18.0 was used for all statistical analyses.

\section{RESULTS}

Assumptions of homogeneity of variance were violated for all DERS subscales, with the exception of lack of emotional awareness (Awareness). In order to correct for violations of homogeneity of variance, Welch $F$-ratio is reported (Table 1).

\subsection{Demographic Variables}

No significant differences were demonstrated between groups with regards to age $F(3179)=0.836, p=0.476$ (HC: $41.36 \pm 11.51$; BD: $44.90 \pm 11.65$; Unipolar Depression: $44.12 \pm 11.71$; Anxiety: $43.73 \pm 10.01$ ) or gender composition $\chi^{2}(3)=5.80, p=0.122$. The proportion of individuals completing secondary and tertiary education were higher among the $\mathrm{HC}$ and $\mathrm{BD}$ groups compared to the Unipolar Depression and Anxiety groups, with a higher proportion of HC participants completing tertiary education than all of the clinical groups. A higher proportion of individuals from the Unipolar Depression 
Table 1. Highest level of educational attainment for each group.

\begin{tabular}{lcccccccc}
\hline \multirow{2}{*}{ Education Level } & $\begin{array}{c}\text { Healthy } \\
\text { Control }\end{array}$ & \multicolumn{2}{c}{$\begin{array}{c}\text { Bipolar } \\
\text { Disorder }\end{array}$} & Depression & Anxiety \\
\cline { 2 - 9 } & $n$ & $\%$ & $n$ & $\%$ & $n$ & $\%$ & $n$ & $\%$ \\
\hline $\begin{array}{l}\text { Did not complete } \\
\text { year 10 }\end{array}$ & 0 & 0 & 2 & 4 & 3 & 6 & 1 & 2 \\
Secondary Education & 13 & 27 & 10 & 22 & 5 & 10 & 7 & 14 \\
Tertiary Education & 32 & 67 & 17 & 35 & 7 & 14 & 10 & 20 \\
Trade Certificate & 2 & 4 & 4 & 8 & 8 & 16 & 8 & 16 \\
Diploma & 1 & 2 & 4 & 8 & - & - & - & - \\
Other & - & - & 1 & 2 & - & - & - & - \\
Missing & - & - & 10 & 20 & 27 & 54 & 24 & 48 \\
\hline
\end{tabular}

and Anxiety groups reported a trade certificate as their highest level of education compared to the BD and HC groups (see Table 1 for details).

\subsection{Emotion Regulation Variables}

Significant differences between groups were reported for all DERS subscales and total DERS (Table 2). Three sets of results were observed. First, inspection of DERS total scores showed that the three clinical groups (BD, depression, and anxiety) reported significantly greater overall emotional dysregulation compared to the healthy control group, with no significant difference in emotion dysregulation between clinical groups. This overall pattern (see Figure 1) was repeated for the specific domains of difficulty engaging in goal directed behaviour (Goals), impulse control difficulties (Impulse), and limited access to emotion regulation strategies (Strategies). All three clinical groups reported significantly greater dysregulation than the HC group for each of these domains, with no significant difference between clinical groups. Second, the Unipolar Depression and Anxiety groups reported significantly greater emotional dysregulation for the domains of non acceptance of emotions (Non-Acceptance), and lack of emotional clarity (Clarity) compared to both the BD and HC groups. Further, the BD group reported significantly greater emotion regulation difficulties for these two subscales compared to the HC group. Third, significant difficulties in regulating emotional Awareness was found for the Unipolar Depression and Anxiety groups compared to the HC group. There were no significant differences in lack of emotional Awareness between the BD and the HC groups, or between the BD and Anxiety groups.

\section{DISCUSSION}

This research aimed to establish an emotional regulation (ER) profile of people with euthymic Bipolar Disorder (BD) and compare it to groups of people diagnosed with euthymic Unipolar Depression, euthymic Anxiety disorder as well as a Healthy Control (HC) group. To this end, the Difficulties in Emotion Regulation Scale (DERS) [9] was used. This scale was favoured given its focus on response tendencies, which makes it a more clinically relevant measure opposed to more pure cognitive or processing approaches to the study of emotions in $\mathrm{BD}$.

Three domains of emotion regulation difficulties appeared to best distinguishable between clinical groups: 1) difficulties accepting emotional responses (Non-Acceptance), 2) emotional clarity (Clarity), and 3) emotional awareness (Awareness). Compared to the Unipolar Depression and Anxiety groups, emotion regulation abilities among the BD group were significantly less compromised with regards to acceptance (Non-Acceptance) and understanding of emotional responses (Clarity). However, some deficits in these emotion regulation abilities were evident when compared to the HC group. On particular interest, our BD sample exhibited similar patterns of ER to the Anxiety group and the HC group for the Awareness subscale. Awareness of emotions in anxiety has already been thoroughly studied [20]. In fact it could be argued that in anxiety, hypervigilance of emotions (fear in particular), leads to more profound dysfunction, if appropriate strategies are not utilised. The Anxiety group was not significantly different from the BD group on this measure but still below the HC group. Anxiety and BD participants appear to share a good level of emotional awareness. Perhaps activation of emotions like fear and a state of elation that characterise anxiety and BD respectively tend to create sufficient frequency and intensity to positively impact on self-reported awareness. Depression and its characteristic emotional bluntness on the other hand could explain the deflated scores on the awareness subscale amongst the Unipolar Depression group here. It appears in any case, that awareness of emotions is not a necessary variable to explain emotion dysregulation. Perhaps the frequency and intensity of emotional experiences discussed for Awareness also applies to Clarity and Non-Acceptance, but this increased exposure does not give them enough acceptance and clarity to reach levels comparable to the normal population. In other words, lack of acceptance of emotional experiences, although more developed than other psychopathologies, still indicates vulnerabilities that might play part in the development of episodes.

However, a caveat seems important here as there might be some psychometric issues with the awareness subscale of the DERS that warrant further refinement. For example, and it has been found to be the only subscale poorly correlated with five of the six clinical variables associated with emotion dysregulation (Non-Acceptance, Goals, Impulsivity, Awareness, Clarity, Strategies) [21]. 
Table 2. Means and standard deviations of difficulties in emotion regulation scale (DERS) for healthy control, bipolar disorder, Unipolar Depression, and Anxiety groups, and results of Welch $F$ statistic.

\begin{tabular}{|c|c|c|c|c|c|c|c|c|c|}
\hline \multirow{2}{*}{ DERS } & \multicolumn{2}{|c|}{ Healthy Control } & \multicolumn{2}{|c|}{ Bipolar Disorder } & \multicolumn{2}{|c|}{ Depression } & \multicolumn{2}{|c|}{ Anxiety } & \multirow[t]{2}{*}{$F$} \\
\hline & $M(n=48)$ & $S D$ & $M(n=48)$ & $S D$ & $M(n=50)$ & $S D$ & $M(n=50)$ & $S D$ & \\
\hline Total & 63.68 & 11.69 & 93.42 & 22.59 & 108.24 & 22.58 & 105.52 & 29.65 & $70.14^{* * *}$ \\
\hline Non-accept & 10.30 & 3.50 & 15.08 & 5.71 & 18.28 & 6.35 & 18.14 & 6.12 & $31.01^{* * *}$ \\
\hline Goals & 11.55 & 4.17 & 16.71 & 4.73 & 17.59 & 3.97 & 17.24 & 5.52 & $20.52^{* * *}$ \\
\hline Impulse & 8.68 & 1.91 & 13.75 & 4.80 & 15.67 & 4.95 & 16.14 & 6.44 & $49.03^{* * *}$ \\
\hline Awareness & 13.38 & 4.03 & 15.46 & 4.97 & 18.78 & 4.90 & 17.78 & 5.06 & $14.22^{* * *}$ \\
\hline Strategies & 10.38 & 2.80 & 19.15 & 6.51 & 21.46 & 6.17 & 20.91 & 8.41 & $67.44^{* * *}$ \\
\hline Clarity & 9.38 & 2.41 & 13.27 & 4.84 & 16.86 & 4.17 & 15.96 & 5.52 & $50.55^{* * *}$ \\
\hline
\end{tabular}

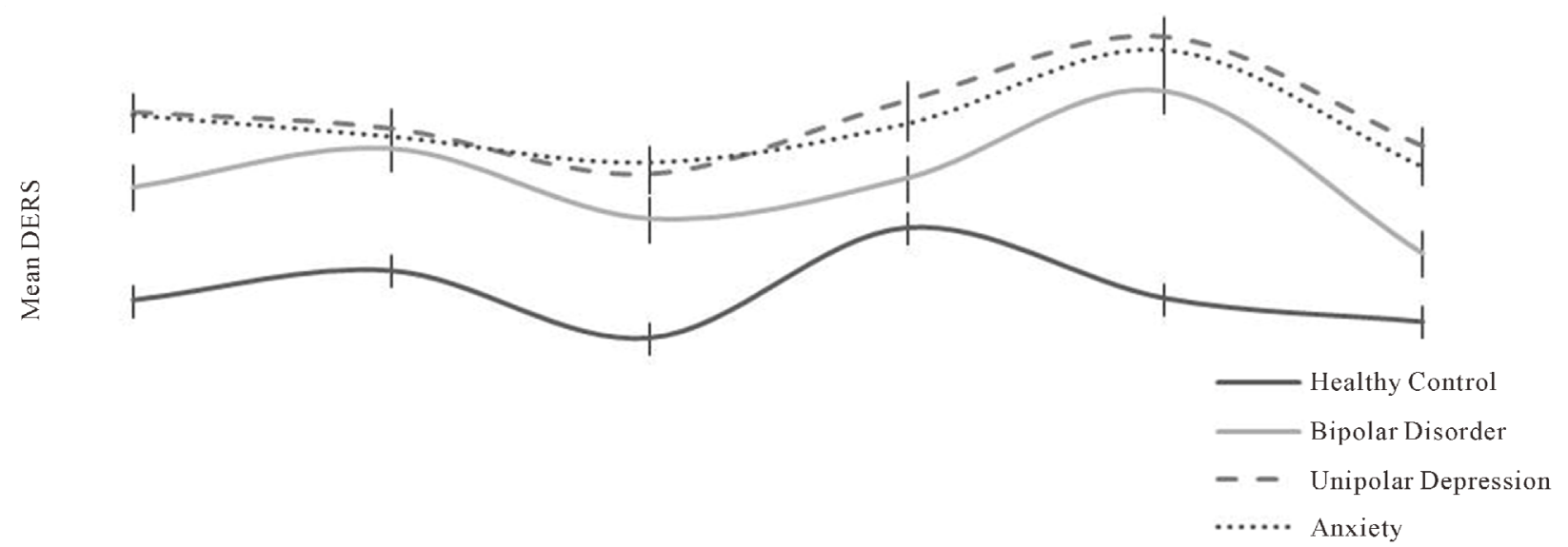

Figure 1. Mean DERS scores for each clinical group compared to healthy controls; Note: Error bars refer to the standard error of the mean (SEM).

The current study identified a number of domains of emotion regulation difficulties that were shared among the three clinical groups (Unipolar Depression, Anxiety, and $\mathrm{BD}$ ), lending itself to a transdiagnostic interpretation. A transdiagnostic approach focuses on the commonalities observed in different psychological disorders, instead of the differences, and this has recently attracted considerable attention in the literature [22-26]. These commonalities could be seen as coincidental by-products of the disorders or, more importantly, as bringing to the fore a causal relationship between putative transdiagnostic mechanisms and psychopathologies. ER appears to be a good candidate for a transdiagnostic analysis of psychopathology as some sort of dysregulation of emotions features in all psychiatric diagnoses $[27,28]$. Specifically, the current study identified commonalities in emotion regulation difficulties between clinical groups in the areas of: Goals, Impulsivity and Strategies. This suggests that irrespective of a diagnosis of Unipolar Depression, Anxiety or BD, the participants in the present study had difficulties engaging in goal directed behaviours when distressed; difficulties modulating their first responses, and show a degree of impulsivity; and they have difficulties accessing constructive coping strategies. These three dimensions of ER seem enough to place them at a disadvantage when facing emotionally challenging situations. In particular, impulsivity and difficulties in engaging in goals were related to the ability to control behaviour (rather than emotions) in emotional episodes in Gratz and Roemer [9] normalisation study of the DERS and were significantly related to clinical outcomes, suggesting that these particular dimensions warrant clinical attention. Our findings support this suggestion in that the three different diagnostic groups behave similarly in these subscales and might therefore be a relevant target for a trans-therapeutic style of intervention.

The complexity observed in our functional approach to ER in participants with $\mathrm{BD}$, to some extent mirrors laboratory findings of emotional processing in $\mathrm{BD}$. A review of the literature [29] indicated that BD patients appear to retain emotional processing (EP) deficits during euthymia, but the picture is complex and includes at times 
marked and other times mild or absent deficits in subcomponents of EP. For example, the review noted that in the area of facial emotional processing in euthymic BD, 12 studies reported no deficits, six studies reported deficits and two studies reported enhanced facial emotion recognition abilities. Similar mixed findings were reported in the area of mood induction, affective attention, emotional memory, auditory processing of emotional information, and affective Theory of Mind [29].

Contradictory findings in several domains pertinent to the emotional phenomena in BD could arise from the fact that when we study a particular diagnosis (e.g., using the Diagnostic and Statistical Manual of Mental Disorders forth revision: DSM-IV) and are focusing on one particular construct (e.g., emotion recognition, or neural substrates, or emotion regulation, etc.). Therefore, we are bound to detect occurrences that might not be specific to any one diagnosis. Additionally, abstract constructs like ER, or emotional processing, appear multifactorial and therefore merit investigations that consider their subcomponents, and these subcomponents could cut across diagnostic taxonomies with various permutations. This discussion is relevant to the clinical domain in that current adjunct psychological interventions for $\mathrm{BD}$ appear to benefit outcome in therapy for BD [30]. For example, adjunct therapies often focus on adherence to medication, psychosocial aspects, sleep hygiene, early warning signs and cognitive and behavioural strategies. Perhaps, integrating emotion regulation techniques into this body of strategies would enhance efficacy, particularly in the areas of goals, impulsivity and strategies, and to a lesser extent in the area of non-acceptance, clarity and awareness. Traditionally this domain has been central to therapies for Borderline Personality Disorders but we suggest that this would aid outcomes in therapy for unipolar depression, anxiety and BD and thus a transdiagnositc approach to therapy appears prudent.

There are some limitations to the present study. Both Bipolar types I and II were included in the study. All BD participants were also prescribed medication, which could have impacted on their impulsivity and awareness (particularly in those taking mood stabilisers and benzodiazepines). Although we argue that by including participants with BD type I and II and participants on medication, we increased the ecological validity of our findings, we also acknowledge the confounding potential these variables represent and hence, we are currently extending this research to the mediating effects of medication and diagnostic category. Future research may go beyond the DERS as a measure of ER and could control for the type of emotions that are more likely to lead to regulatory deficits as there might be a more powerful effect when subjects face negative versus positive, basic versus complex motions, and so on. Finally simultaneous comparison with multiple diagnoses (e.g., anxiety, depression, Borderline, etc.) would add clarity to a transdiagnostic map of emotion regulation that interconnects diagnostic groups and focuses on strategies to regulate challenging emotional scenarios and thus directly inform therapeutic interventions.

\section{CONCLUSIONS}

In conclusion, the present study contributed to the creation of an ER profile for people with BD and compared it to that of people diagnosed with Unipolar Depression, Anxiety and a Healthy Control group. Results suggested that the DERS and its subscales yield a useful transdiagnostic map of ER abilities that interconnect and divide traditional taxonomies into ER based abilities. In particular, when faced with emotional situations, ER in people with euthymic BD appears to be characterised by a normal level of emotional awareness, with difficulties engaging in goal directed behaviour, impulsivity, and limited access to useful strategies. Further, the ability to accept and understand emotions appears to be less compromised among people with BD compared to people with Unipolar Depression and Anxiety. However, deficits are still evident compared to Healthy Control participants.

This profile might help enrich extant adjunct psychological interventions for BD by enlisting ER strategies normally reserved for Borderline Personality Disorder programs, with the aim of decreasing the relapse rate that characterises BD. Further research would be needed to refine these findings.

\section{REFERENCES}

[1] Lam, D., Hayward, P., Watkins, W. and Sham, P. (2005) Outcome of a two-year follow-up of a cognitive therapy of relapse prevention in bipolar disorder. The American Journal of Psychiatry, 162, 324-329. http://dx.doi.org/10.1176/appi.ajp.162.2.324

[2] Scott, J., Paykel, E.S., Morriss, R., Bentall, R., Kinderman, P., Johnson, T., et al. (2006) Cognitive behavioural therapy for severe and recurrent bipolar disorders. The British Journal of Psychiatry, 188, 313-320. http://dx.doi.org/10.1192/bjp.188.4.313

[3] Simon, G.E., Ludman, E.J., Unutzer, J., Bauer, M.S., Operskalki, B. and Rutter, C. (2005) Randomised trial of a population-based care program for people with bipolar disorder. Psychological Medicine, 35, 13-24. http://dx.doi.org/10.1017/S0033291704002624

[4] Ball, J.R., Mitchell, P.B., Corry, J.C., Skillecom, A. and Malhi, G.S. (2006) A randomized controlled trial of cognitive therapy for bipolar disorder: Focus on long-term change. Journal of Clinical Psychiatry, 67, 277-286. http://dx.doi.org/10.4088/JCP.v67n0215

[5] Fisher, L., Goldney, R.D., Dal Grande, E.D., Taylor, A.W. 
and Hawthorne, G. (2007) Bipolar disorders in Australia a population-based study of excess costs. Social Psychiatry and Psychiatric Epidemiology, 42, 105-109. http://dx.doi.org/10.1007/s00127-006-0133-4

[6] Johnson, S.L., Gruber, J. and Eisner, L. (2007) Emotion in bipolar disorder. In: Rottenberg, J.E. and Johnson, S.L., Eds., Emotion and Psychopathology: Bridging Affective and Clinical Science, American Psychological Association, Washington DC.

[7] Gross, J.J. and Munoz, R.F. (1995) Emotion regulation and mental health. Clinical Psychology: Science and Practice, 2, 151-164. http://dx.doi.org/10.1111/j.1468-2850.1995.tb00036.x

[8] Cole, P.M., Michel, M.K. and Teti, L.O. (1994) The development of emotion regulation and dysregulation: A clinical perspective. Society for Research in Child Development, 59, 73-100. http://dx.doi.org/10.2307/1166139

[9] Gratz, K.L. and Roemer, L. (2004) Multidimensional assessment of emotion regulation and dysregulation: Development, factor structure, and initial validation of the difficulties in emotion regulation scale. Journal of Psychopathology and Behavioral Assessment, 26, 41-54. http://dx.doi.org/10.1023/B:JOBA.0000007455.08539.94

[10] Ehring, T., Fischer, S., Schnulle, J., Bosterling, A. and Tuschen-Caffier, B. (2008) Characteristics of emotion regulation in recovered depressed versus never depressed individuals. Personality and Individual Differences, 44, 1574-1584. http://dx.doi.org/10.1016/j.paid.2008.01.013

[11] Ehring, T., Tuschen-Caffier, B., Schnulle, J., Fischer, S. and Gross, J.J. (2010) Emotion regulation and vulnerability to depression: Spontaneous versus instructed use of emotion suppression and reappraisal. Emotion, 10, 563572. http://dx.doi.org/10.1037/a0019010

[12] Mennin, D.S., Heimberg, R.G., Turk, C.L. and Fresco, D.M. (2005) Preliminary evidence for an emotion dysregulation model of generalized anxiety disorder. Behaviour Research and Therapy, 43, 1281-1310. http://dx.doi.org/10.1016/j.brat.2004.08.008

[13] Salters-Pedneault, K., Roemer, L., Tull, M.T. and Mennin, D.S. (2006) Evidence of broad deficits in emotion regulation associated with chronic worry and generalised anxiety disorder. Cognitive Therapy and Research, 30, 469480. http://dx.doi.org/10.1007/s10608-006-9055-4

[14] Tull, M.T. and Roemer, L. (2007) Emotion regulation difficulties associated with the experience of uncued panic attacks: Evidence of experiential avoidance, emotional nonacceptance, and decreased emotional clarity. Behaviour Therapy, 38, 378-391. http://dx.doi.org/10.1016/j.beth.2006.10.006

[15] Gruber, J., Harvey, A.G. and Gross, J.J. (2012) When trying is not enough: Emotion regulation and the effort-success gap in bipolar disorder. Emotion, 12, 9971004. http://dx.doi.org/10.1037/a0026822

[16] Gratz, K.L. and Gunderson, J.G. (2006) Preliminary data on an acceptance-based emotion regulation group intervention for deliberate self-harm among women with bor- derline personality disorder. Behaviour Therapy, 37, 2535. http://dx.doi.org/10.1016/j.beth.2005.03.002

[17] Fox, H.C., Axelrod, S.R., Paliwal, P., Sleeper, J. and Sinha, R. (2007) Difficulties in emotion regulation and impulse control during cocaine abstinence. Drug and Alcohol Dependence, 89, 289-301.

http://dx.doi.org/10.1016/j.drugalcdep.2006.12.026

[18] Hamilton, M. (1980) Rating depressive patients. Journal of Clinical Psychiatry, 41, 21-24.

[19] Young, R.C., Biggs, J.T., Ziegler, V.E. and Meyer, D.A. (1978) A rating scale for mania: Reliability, validity and sensitivity. The British Journal of Psychiatry, 133, 429435. http://dx.doi.org/10.1192/bjp.133.5.429

[20] Mennin, D.S., McLaughlin, K.A. and Flanagan, T.J. (2009) Emotion regulation deficits in generalized anxiety disorder, social anxiety disorder, and their co-occurrence. Journal of Anxiety Disorders, 23, 866-871. http://dx.doi.org/10.1016/j.janxdis.2009.04.006

[21] Weinberg, A. and Klonsky, E.D. (2009) Measurement of emotion dysregulation in adolescents. Psychological Assessment, 21, 616-621. http://dx.doi.org/10.1037/a0016669

[22] Mansell, W., Harvey, A., Watkins, E. and Shafran, R. (2009) Conceptual foundations of the transdiagnostic approach to CBT. Journal of Cognitive Psychotherapy, 23, 6-19. http://dx.doi.org/10.1891/0889-8391.23.1.6

[23] Kring, A.M. and Sloan, D.S. (2009) Emotion regulation and psychopathology: A transdiagnostic approach to etiology and treatment. Guilford Press, New York.

[24] Harvey, A.G., Watkins, E.R., Mansell, W. and Shafran, R. (2004) Cognitive behavioural processes across psychological disorders: A transdiagnostic approach to research and treatment. Oxford University Press, Oxford.

[25] Ehring, T. and Watkins, E.R. (2008) Repetitive negative thinking as a transdiagnostic process. International Journal of Cognitive Therapy, 1, 192-205.

http://dx.doi.org/10.1521/ijct.2008.1.3.192

[26] Nolen-Hoeksema, S. and Watkins, E.R. (2011) A heuristic for developing transdiagnostic models of psychopathology, explaining multifinality and divergent trajectories. Perspectives on Psychological Science, 6, 589-609. http://dx.doi.org/10.1177/1745691611419672

[27] Rottenberg, J. and Johnson, S.L. (2007) Emotion and psychopathology: Bridging affective and clinical science. APA Books, Washington DC.

[28] Kring, A.M. (2008) Emotion disturbances as transdiagnostic processes in psychopathology. In: Lewis, M., Haviland-Jones, J.M. and Barrett, L.F., Eds., Handbook of Emotion, 3rd Edtion, Guilford Press, New York, 691-705.

[29] Mercer, L. and Becerra, R. (2012) A unique emotional processing profile of euthymic bipolar disorder? A critical review. Journal of Affective Disorders, 146, 259-309.

[30] American Psychiatric Association (2002) Practice guideline for the treatment of patients with bipolar disorder (revision). The American Journal of Psychiatry, 159, 150 . 